\title{
Erratum to: NanoCAGE: A Method for the Analysis of Coding and Noncoding 5'-Capped Transcriptomes
}

Stéphane Poulain, Sachi Kato, Ophélie Arnaud, Jean-Étienne Morlighem, Makoto Suzuki, Charles Plessy, and Matthias Harbers

\section{Erratum to:}

Chapter 4 in: Sara Napoli (ed.), Promoter Associated RNA: Methods and Protocols, Methods in Molecular Biology, vol. 1543, DOI 10.1007/978-1-4939-6716-2_4

The original version of the book was inadvertently published without incorporating the author's proof corrections. The chapter has now been corrected and approved by the author. 